\title{
A Novel Approach for Quality Control System using Sensor Fusion of Infrared and Visual Image Processing for Laser Sealing of Food Containers
}

\author{
A. Al-Habaibeh*, F. Shi, N. Brown, D. Kerr, M. Jackson and R. M. Parkin ${ }^{* *}$ \\ *School of Computing and Technology, The Nottingham Trent University, \\ Burton Street, Nottingham, NG1 4BU,UK \\ Email: Amin.Al-Habaibeh@ntu.ac.uk \\ Mechatronics Research Centre, Wolfson School of Mechanical and Manufacturing \\ Engineering, Loughborough University, Loughborough, LE11 3TU, UK \\ www.mechatronics.org.uk
** IENSYS Ltd, Intelligent Engineering Systems, Holywell Building, Holywell Way, Loughborough, LE11 3UZ, UK www.iensys.com

\begin{abstract}
This paper presents a new mechatronic approach of using infrared thermography combined with image processing for the quality control of a laser sealing process for food containers. The suggested approach uses an on-line infrared system to assess the heat distribution within the container seal in order to guarantee the integrity of the process. Visual image processing is then used for quality assurance to guarantee optimum sealing. The results described in this paper show examples of the capability of the condition monitoring system to detect faults in the sealing process. The results found indicate that the suggested approach could form an effective quality control and assurance system.
\end{abstract}

Keywords: Laser sealing, image processing, infrared, condition monitoring, quality control.

\section{Introduction}

Human inspection is currently the common method in the food industry to ensure the integrity of plastic food containers sealed by a conventional thermal/pressure process. The seal quality is usually tested by an off-line sampling process. In order for food packs to gain longer shelf life and for companies to reduce wastage and increase production efficiency, a reliable automated system of condition monitoring of the sealing process is needed. The laser sealing process described in this paper is a noncontact sealing system for lidding films, as used in dual-ovenable trayed and ambient ready meals [1]. The method uses a beam-steered laser to seal the product. This allows high flexibility in changing product lines without having to change the hardware (i.e. heated sealing head). This reduces the down-time for set-up and the capital investment in tooling. The quality assurance system previously described in $[2,3]$ depends on visual image processing of the containers after the welding process has finished. An off-line (destructive) tensile test can also be performed to test the quality of the sealing.

In order to provide an autonomous quality control system as well as a quality assurance system, this paper investigates the use of infrared imaging data combined with white light image processing. Using this sensor fusion approach, the wastage of 
rejected food containers can be reduced or eliminated and the quality of the sealing process and the product can be guaranteed.

Many research papers have been published in the field of laser welding of plastics [47]. Condition monitoring of laser welding has also been the focus of much recent research work [8-10]. Conventional polymer welding processes are quite different from the laser sealing technique used in this work, in that very little mechanical pressure is applied to the joint and no laser absorbent interface material is added. An understanding of heat absorption at the joint interface is thus crucial to the optimisation of the sealing process. This paper presents a novel approach of combining infrared image processing with visual image processing for quality control and assurance of laser sealing of food containers.

\section{Sensor Fusion of infrared and visual image processing}

A schematic diagram of the proposed system is shown in Figure 1. The filled containers (trays) are located below the lidding film and the laser source. The film is stretched flat using tension across rollers and a spreading bar and is held in close contact with the upper surface of the tray. Based on the tray geometry, film and tray material type, an optimised laser beam (focus/power) scans the tray and lidding film interface for complete sealing. The infrared imager captures the on-line heat distribution of the scanned tray during the sealing process as well as during the first few seconds of the cooling process. The system then compares the thermographical behaviour of the current tray with the expected normal sealing process. If the thermographical behaviour is normal, then the tray is moved to the visual image processing system for seal integrity confirmation. However, if the infrared data indicates any abnormal behaviour, the data is compared with previous faults/problems and the laser delivery and film-handling systems may be adjusted accordingly. The infrared camera has also the advantage of monitoring the actual power of the laser system; therefore, by combining the infrared data with the visual image processing, the reason for any incomplete sealing will be identified. 


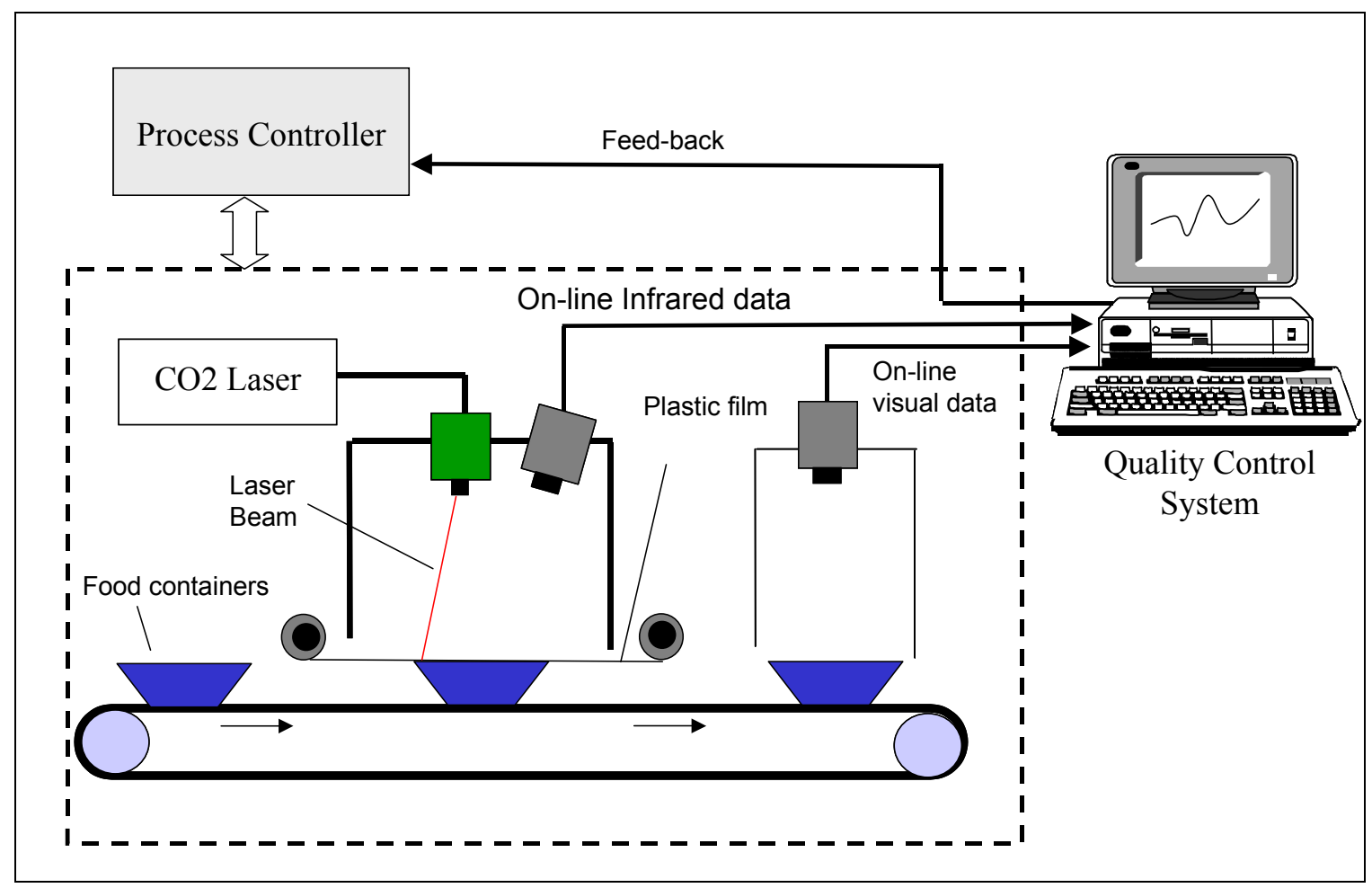

Figure 1: A schematic diagram for the complete sealing and monitoring system

As shown in Figure 1, the computer system monitors the data of both systems and can thus gather any significant information for a self-learning process.

The advantages of using an infrared camera are that it:

1. Monitors the power of the laser system either by monitoring the peak temperature of the sealed trays or by establishing a regular diagnostic system (i.e. heating a special component for a specific amount of time and measuring its temperature using the infrared system).

2. Monitors the shape of the sealing patterns.

3. Monitors the cooling process cycle-time to establish the quality of the seal.

4. Can reveal the effect of fume extraction or other ambient conditions on the cooling process (in cases where these cause inconsistency in the quality of the weld).

\section{The Experimental Work}

The experimental work was performed to test the visual and infrared monitoring and quality control strategy. Figure 2 illustrates the experimental set-up, including the visual and infrared imaging system. The laser system used for the experimental work was a 50W RF excited Carbon Dioxide laser with a wavelength of $10.6 \mu \mathrm{m}$ (infrared). The sealing rig is designed to seal any tray shape using the non-contact laser sealing process. The test rig is designed for container handling, film handling, laser scanning and can be easily integrated with a conveyor system to develop a complete industrial system. The laser system is used for the sealing processes as well as for cutting the extra sealing film from around the tray using an automatic re-focusing system. The laser scanning is done using galvanometric mirrors, an integrated motor, and a pneumatic system control. It takes about 1 second to scan the container using the laser system. However, this time can be shortened using a faster scanning system. 


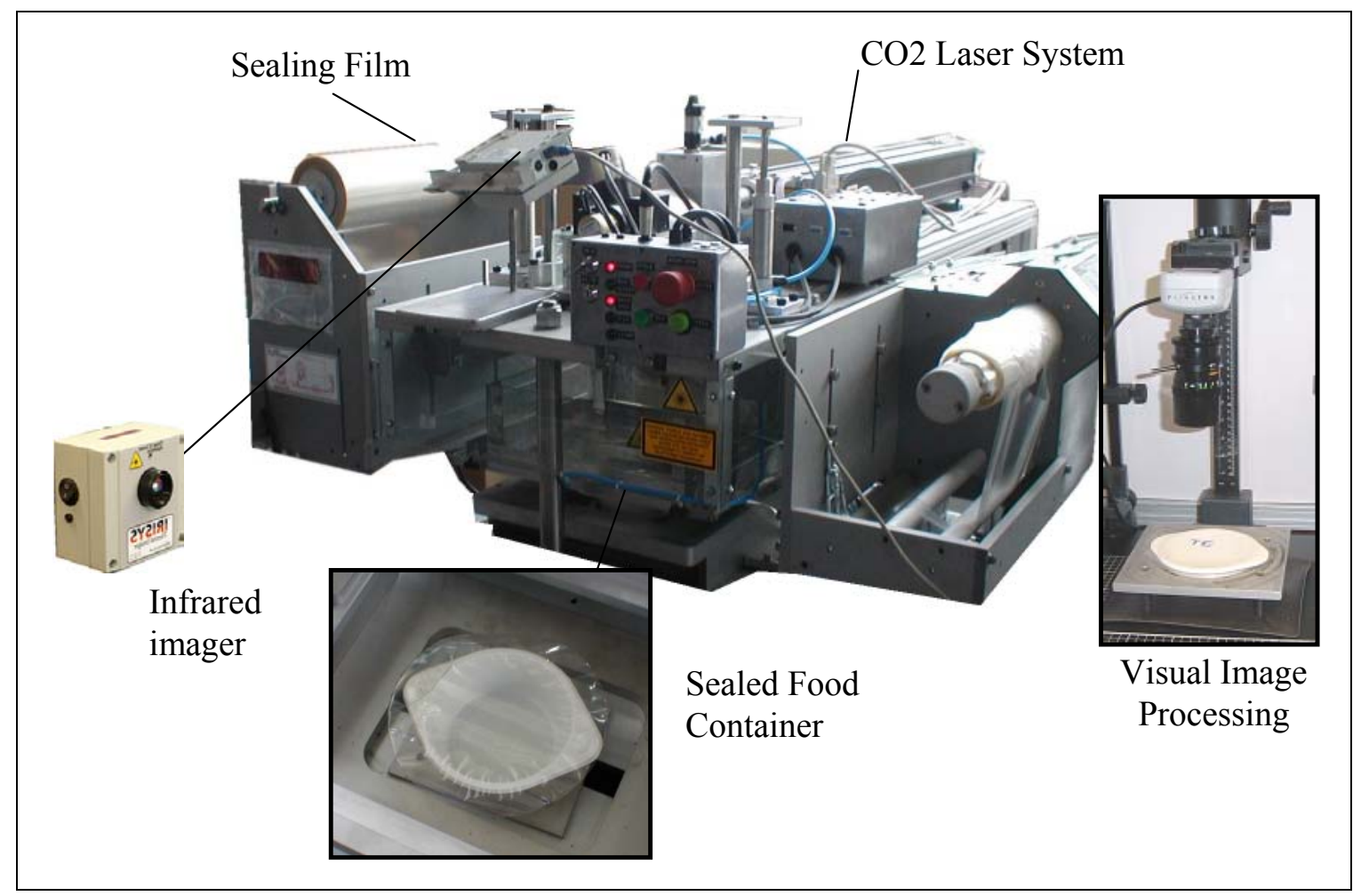

Figure 2: Experimental set-up including the infrared camera, visual camera, laser system and the sealing test rig.

The infrared imager used is a low-resolution, low-cost IRISYS multi-point radiometer type IRI1002 [11], fitted with a germanium focussing lens. The spectral response of the device is between 8 and $14 \mu \mathrm{m}$ and without optical filtering; it is therefore sensitive to both reflected/scattered laser light and re-radiated heat. This is an important consideration when using the device to monitor sealing performance and is dealt with later in section 4. The IRI 1002 Radiometer is housed in a robust metal case. The imaging optics, detector, drive electronics and optical modulator are all enclosed in this case. Data output is provided in RS232 to the monitoring PC. The original resolution of the imager is $16 \times 16$ pixels but it can be interpolated to $128 \times 128$ to provide an improved visual interpretation. The visual monitoring system used is composed of a firewire CCD camera of high resolution up to $1280 \times 1024$ pixels, a zoom lens and various lighting devices. The system also has a built-in extraction and filtration unit to remove any fumes generated by the sealing and cutting process. MATLAB software is used for the analysis of the visual and infrared data.

\section{Results and Discussion}

In order to determine whether a laser seal is acceptable, the investigation of both conventionally sealed and laser-sealed food containers has revealed that a good sealing must have:

1. A completely uniform texture of the sealing surface.

2. Sealing peel and push strength within an appropriate range.

3. Zero or minimum contamination around the sealing zone.

4. Sufficient 'hot tack' seal strength to withstand automated handling.

The power and speed of the laser system was optimised by testing the strength of the sealant peel. Then the experimental work was performed to test the capability of the 
infrared system and visual system to detect any abnormalities in the sealing processes or sealing quality.

\subsection{Infrared Image Processing}

Figure 3 presents some of the results obtained during the experimental work, using the infrared imaging system to characterise the integrity of the process. Figure 3-a shows the localised heat caused by the laser beam during the welding process, i.e. with the incident laser beam switched on. The bright white area represents the current position of the laser spot on the seal of a circular tray, whereas the darker areas show radiated heat from the seal periphery. It was found that due to the extensive scattering of infrared radiation during sealing, the pixels in the immediate area of the incident beam are saturated. Therefore, the number of saturated pixels can be used to characterise the strength and shape of the laser spot for a given material, lamination, tray size and incidence angle.

The temperature values shown are relative readings since the focus of the monitoring system is to compare what is "normal" with what is faulty rather than measuring the absolute temperature. Figure 3-b shows a typical heat profile of the normal welding process, taken 5 seconds after switching off the laser beam at seal completion. The same profile is shown in the infrared image of Figure 3-c. It can be seen that the heat distribution is relatively even around the entire tray periphery. An example of a bad seal due to deliberate contamination of the surface between the sealing film and the containers is shown in Figure 3-d. Notice the discontinuity in the infrared profile of Figure 3-d when compared with Figures 3-c and 3-b.

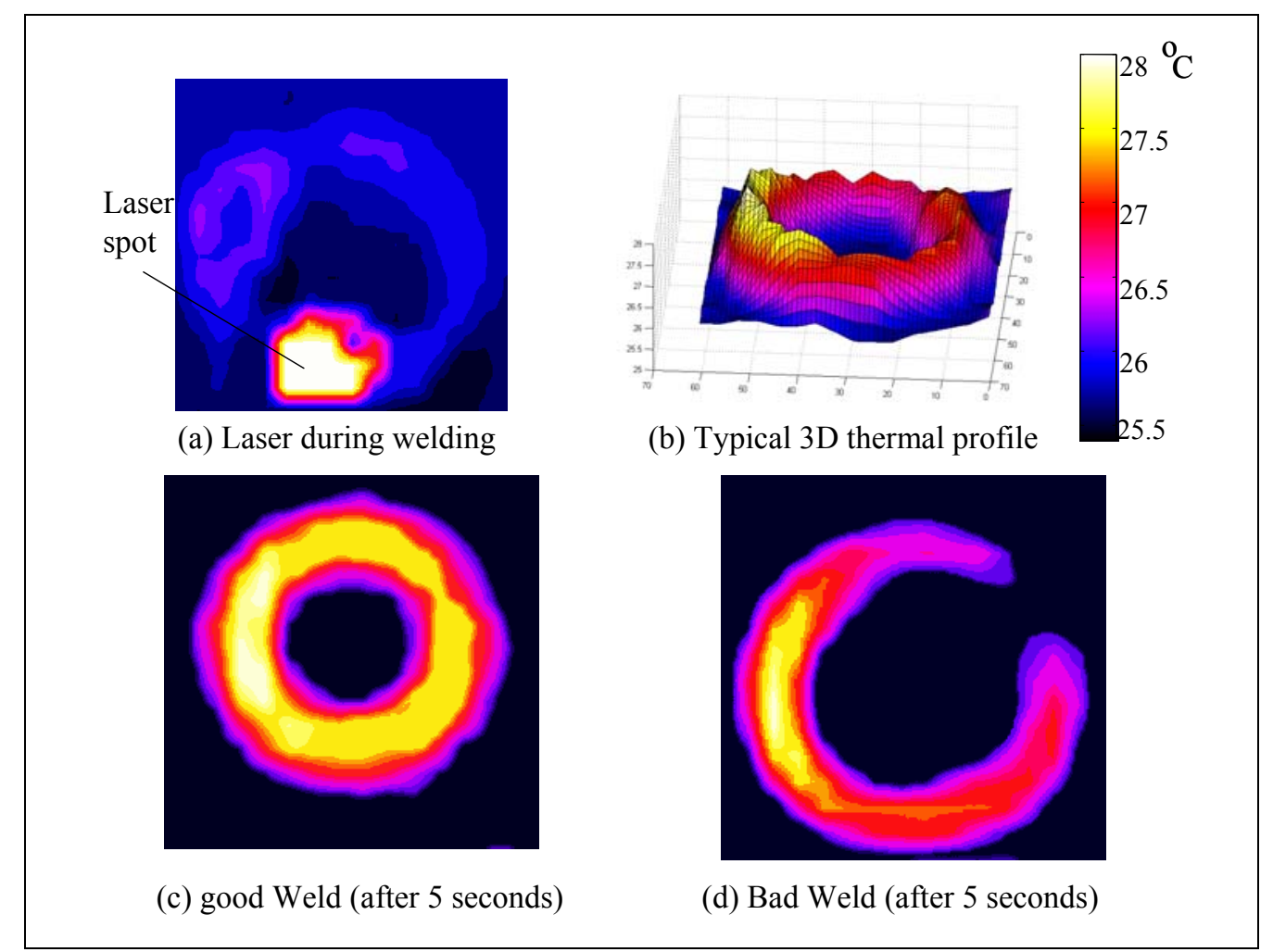

Figure 3: Examples of Infrared results: The tray during laser welding (a), the 3D temperature surface of a normal weld (b), an infrared image of a good weld (c) and an infrared image of a bad weld (d). 
It could be argue that it might not be possible to wait few second after the welding process to monitor the thermal behaviour. However, if the infrared system is moved in the same speed with the container in real industrial environment, such monitoring period should be achievable. One interesting design problem found in the laser sealing system, which was not recognised until the implementation of the infrared sensor, is the influence of the fume extraction device on the heat distribution within the seal. Figure 4 shows the maximum temperature reached by two areas on the tray immediately after the end of the sealing process and the subsequent cooling characteristics. It was found that the fume extraction process, because it is not symmetric, influences the temperature profile. This could vary the sealing strength characteristic in different parts on the sealed circumference. This phenomenon suggests another possible application of the IR sensor in the packaging process. The common industrial practice, after sealing, is to cool rapidly the trays containing hot food before the product is packed. In many cases, this chilling process can considerably weaken the seal. IR monitoring of the rapid cooling of sealed trays could help to prevent poorly sealed products from being delivered to the packaging cycle or even to the customer.

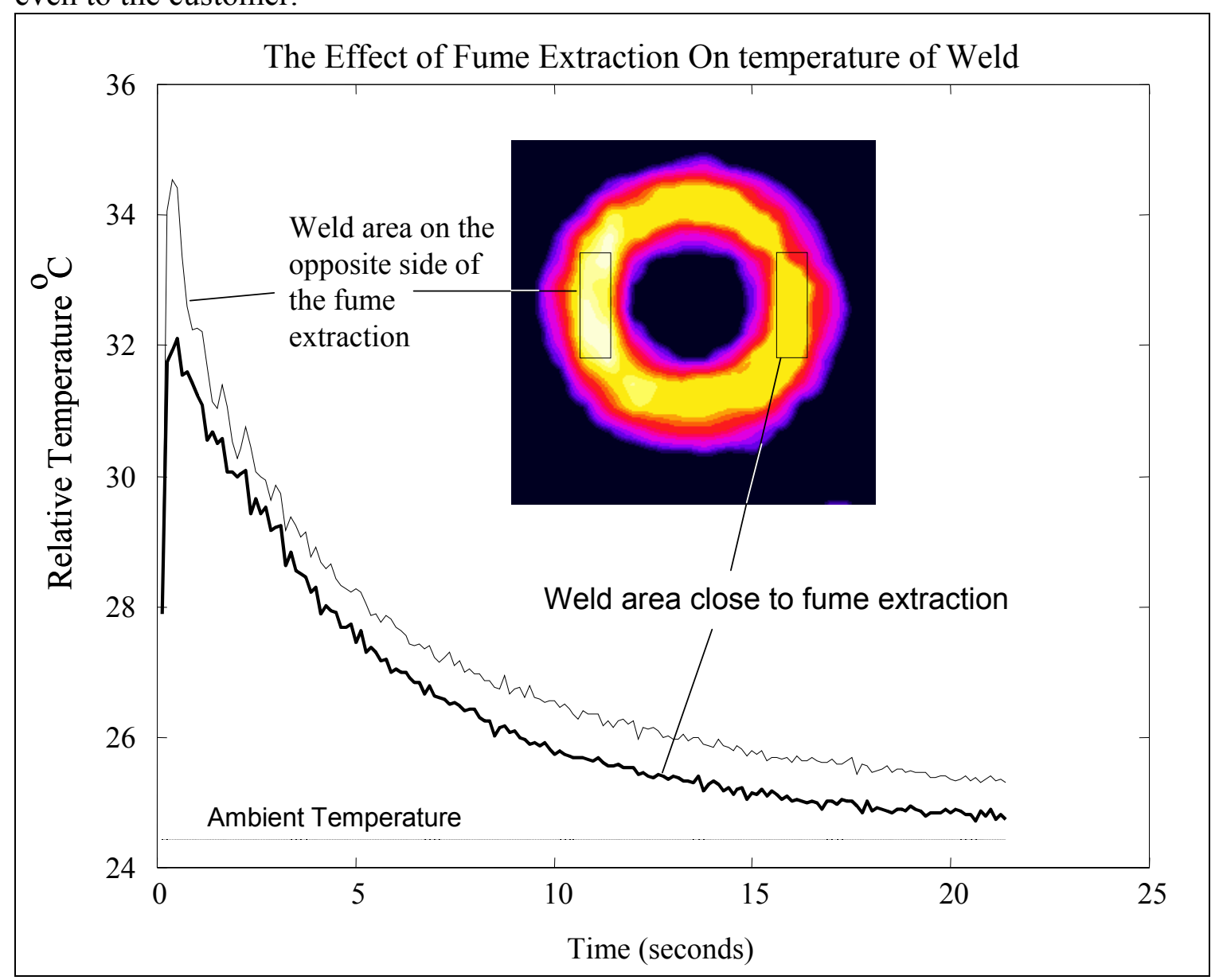

Figure 4: The effect of fume extraction on maximum temperature reached and the subsequent cooling curve.

The low resolution image is found to be sufficient in detecting problems in the thermal pattern for two reasons:

1. The laser system scans the container many times to include a relatively thick welded area. 
2. For normal welding process, the heat is expected to spread even wider and the heat pattern should be easily detected with low resolution imager. The infrared imager has a temperature range between $-10^{\circ} \mathrm{C}$ to $300{ }^{\circ} \mathrm{C}$. Saturation could mean either the temperature has exceeded $300{ }^{\circ} \mathrm{C}$ or the laser radiation generates high intensity to cause the sensor to saturate in the localised area where the beam is operating.

\subsection{Visual Image Processing}

One visual image (1280x1024 pixels) is used to capture the visual data of the container. It has been found by visual testing of images that a good laser seal has a uniform texture when compared with a bad seal [2]. Any non-uniformity of laser sealing usually results from an inappropriate usage of laser power. An algorithm based on grey level variance and thresholding was used to distinguish between a "normal" and "bad" seal. Figure 5-a and Figure 5-b show a good and a bad seal image respectively. Due to space limitations, only a small part of the visual images are presented. The presented results are obtained from transparent films using a white light. Other tests have been performed using red light. It is found that red light gives more glare and less consistent results.

The weld area was automatically segmented using curve fitting based on the Hough transform [12]. The purpose of using Hough transform is to find the coefficients $a, b$ and $\mathrm{c}$ in the parabolic curves described by the equation $y=a x^{2}+b x+c$ to determine the curve coving the seal area. A variance operator was then applied to replace every individual pixel with the variance value of its centred $5 \times 5$ window. A threshold of 80 was then chosen and applied to the variance data within the weld zone. Comparing Figure 5-a with Figure 5-b of the processed images, the good weld zone appears totally black, whereas the bad weld zone still contains 2309 white pixels. Thus, a white pixel count of the thresholded variance can relate directly to seal uniformity and seal quality.

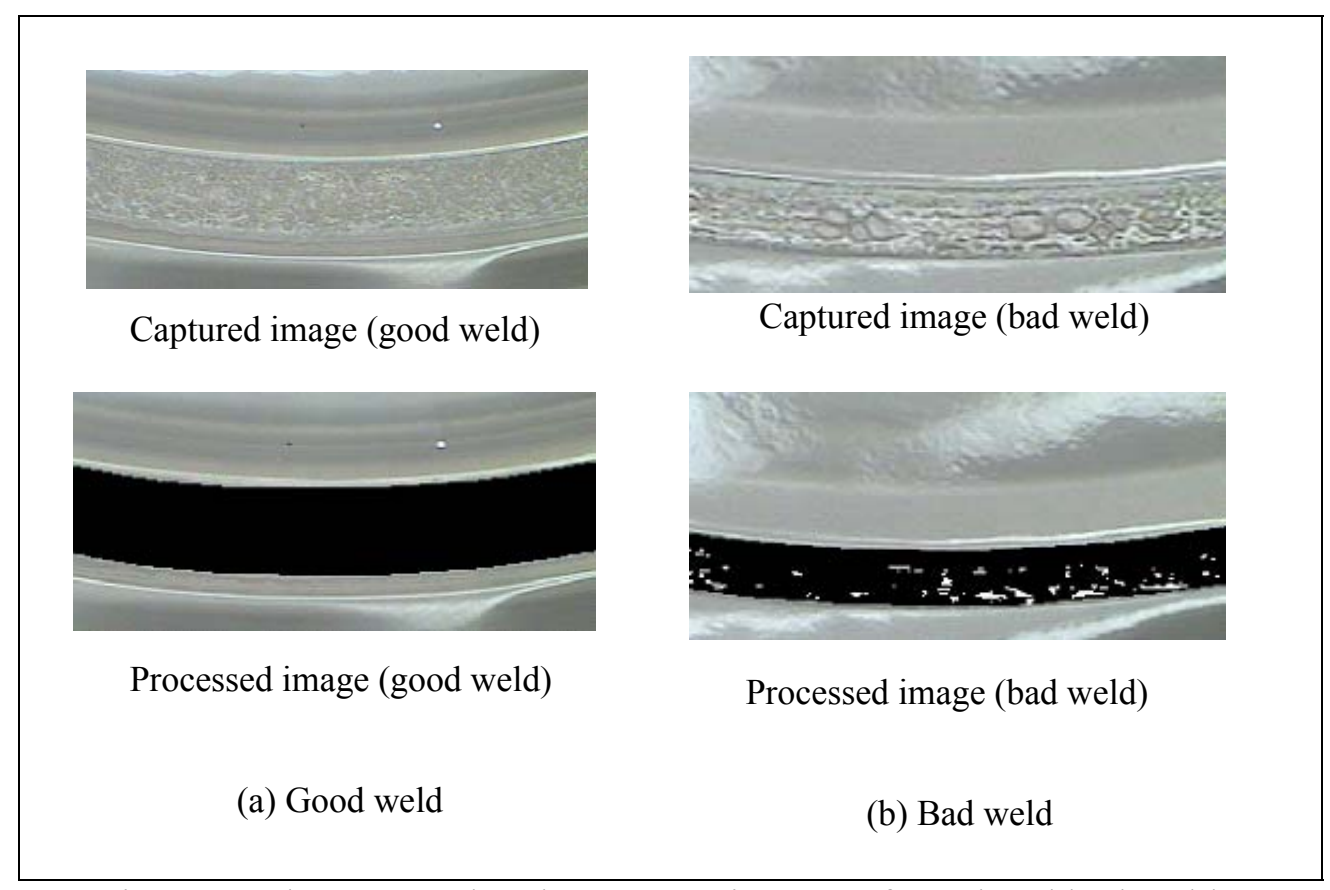

Figure 5: The captured and processes images of good and bad welds. 
The overall processing time of the image is about than $20 \mu$ s depending on the speed of the computer system. The visual image is capable of detecting non-welded areas within about $0.25 \times 0.25 \mathrm{~mm}$. The processing of the infrared data should detect any large, non-welded areas as well as the normal thermal behaviour of the welded area. This should provide good information regarding the general health of the process. The visual image processing is used to detect small areas that is non-welded for two main reasons:

1. As a confirmation of the results obtained from infrared data .

2. In case the container is sealed but the peeling force is small, which makes the container weak for transportation.

\section{Conclusions and Further Work}

This paper has described a novel approach of using sensory fusion of infrared and visual image processing as part of a quality assurance and quality control system for laser sealing of food containers. The developed sealing system provides the flexibility for sealing different shapes and sizes of containers without the need for dedicated hardware. The application of infrared and visual image processing provides a promising system for quality assurance and control. The infrared system is found to be capable of detecting the localised heat pattern, the contamination between the film and the container, and the cooling behaviour of the materials after welding. The visual infrared system provides a quality assurance system for the integrity of the sealed products. Future work will consist of developing the experimental apparatus into an industrial system for sealing of plastic containers.

\section{Acknowledgments}

The authors would like to thank the project industrial partners, Packaging Automation Ltd, GSI Lumonics Ltd, The Recipe Dish Company Ltd, FFP Packaging Solutions Ltd, and RPC Containers Ltd. The project is funded under the DEFRA/LINK Programme Code AFM114, "Intelligent Package Sealing and Inspection System for Semi-rigid Food Containers"[http://www.defra.gov.uk].

\section{References}

[1] Brown N., Kerr D., Jackson M. R., Parkin R. M., "Laser welding of thin polymer films to container substrates for aseptic packaging", Optics and Laser Technology, 2000. 32(2): p. 139-146.

[2] Fangmin Shi, Neil Brown, David Kerr, Mike Jackson and Rob Parkin ,"A machine vision approach to quality inspection of laser welded, semi-rigid food containers", Proceedings ICOM03, International Conference on Mechatronics, Eds. R.M. Parkin, A. Al-Habaibeh, M.R. Jackson, Loughborough, UK, 18th June 2003, pp 371-376, ISBN 1860584209.

[3] Brown, N., Shi, F., Kerr, D., Jackson, M.R., Parkin, R.M. "CO2 Laser Processing of Packaging Films Including Multilayer Laminates Containing Silicon Oxide and Ethylene Vinyl Alcohol Barrier Layers", Proceedings ICOM03, International Conference on Mechatronics, Eds. R.M. Parkin, A. Al-Habaibeh, M.R. Jackson, Loughborough, UK, 18th June 2003, pp 371-376, ISBN 1 860584209 .

[4] Coelho, J.P., M.A. Abreu, and M.C. Pires, High-speed laser welding of plastic films. Optics and Lasers in Engineering, 2000. 34(4-6): p. 385-395. 
[5] J Coelho, J.S., M Pedro,, M Pires. Laser cutting of superposed plastic films. International Conference on Lasers 98. 1998. Tucson, AZ, USA: STS Press.

[6] J Coelho, M.L., M Abreu, M Pires. Influence of Laser Spot Shape on Welding of Thin Thermoplastics. in Laser Materials Processing Conference ICALEO 2000. 2000: Laser Institute of America.

[7] Edward, B.R., Pogreb; Avigdor, Sheshnev; Eugene, Shulzinger; Yelena, Bormashenko; Katzir, Abraham, IR laser radiation induced changes in the IR absorption spectra of thermoplastic and thermosetting polymers. Journal of Optics A: Pure and Applied Optics, 2001. 3: p. 229 - 235.

[8] Kamimuki, K.; Inoue, T.; Yasuda, K.; Muro, M. ; Nakabayashi, T.; Matsunawa, A., "Prevention of welding defect by side gas flow and its monitoring method in continuous wave Nd:YAG laser welding", Journal of Laser Applications, v 14, n 3, (August 2002), ISSN: 1042-346X, pp 136-145.

[9] Zeng, H.; Zhou, Z. D.; Chen, Y. P.; Luo, H.; Hu, X. Y.; Hu, L. J., "Real-time inprocess monitoring of laser welding", Zhongguo Jiguang/Chinese Journal of Lasers, v 28, n 3, (March 2001), ISSN: 0258-7025, p 284-288

[10] Lim, D. -C.; Cho, Y. -B.; Gweon, D. -G., "Robust in-process monitoring of pulsed laser spot welding using a point infrared sensor", Proceedings of the Institution of Mechanical Engineers, Part B: Journal of Engineering Manufacture, v 212, n B3, (1998), ISSN: 0954-4054, p 241-250.

[11] Al-Habaibeh, A. and Parkin, R., "An Autonomous Low-Cost Infrared System for the On-Line Monitoring of Manufacturing Processes Using Novelty Detection" , International Journal of Advanced Manufacturing Technology, 22(3-4), September 2003, pp 249-258, ISSN 0268-3768 .

[12] Sonka, M., Hlavac, V. and Boyle, R. 1993. Image Processing, Analysis and Machine Vision. UK: Chapman \& Hall. 